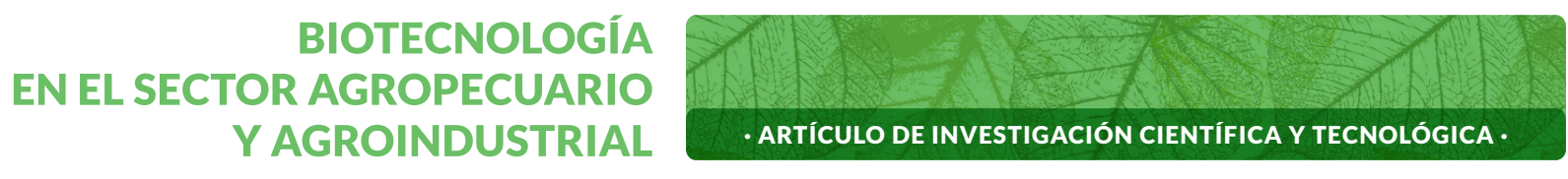

Vol. 19 No 1 · Enero - Junio 2021 · ISSN - 1692-3561 · ISSN-e 1909-9959 · DOI: https://doi.org/10.18684/BSAA(19)206-216

\title{
Ácidos ganodéricos en Ganoderma sp, Ganoderma lucidum y en su sustrato agotado*
}

\author{
Ganoderics acids from Ganoderma sp, \\ Ganoderma lucidum and exhausted substrate
}

\section{Ácidos ganodéricos na Ganoderma sp, Ganoderma lucidum e em seu substrato esgotado}

RAMÍREZ-AYALA, WILDER ${ }^{1}$; GUEVARA-BRAVO, CARLOS-ALBERTO2; RODRÍGUEZ-ESPINOSA, JHON-ALEXANDER ${ }^{3}$

Historial del Artículo

Recibido para evaluación: 22 de Mayo 2019.

Aprobado para publicación: 20 de Octubre 2020

* Título del proyecto de origen: "Compuestos Bioactivos de Ganoderma lucidum, de su sustrato agotado y de Ganoderma silvestre de la zona; obtenidos mediante fluídos supercríticos y analizados por Cromatografía Líquida de Alta Eficiencia (HPLC)". Financiación Universidad del Quindío. Culminación Junio de 2018.

1 Universidad del Quindío, Maestría en Química, Grupo de Investigación en Ciencias Ambientales (INCIAM). Licenciado en Biología y Educación Ambiental, Magister en Química. Armenia, Colombia. https://orcid.org/0000-0001-6988-9483

2 Universidad del Quindío, Maestría en Química, Grupo de Investigación en Ciencias Ambientales (INCIAM). Doctor en Ciencias Químicas. Armenia, Colombia. https://orcid. org/0000-0002-7237-4197

3 Universidad del Quindío, Maestría en Química, Grupo de Investigación en Ciencias Ambientales (INCIAM). Magister en Química. Armenia, Colombia. https://orcid.org/00000001-7445-1662

Correspondencia: inciam@uniquindio.edu.co

Cómo citar este artículo: RAMÍREZ-AYALA, WILDER; GUEVARA-BRAVO, CARLOSALBERTO; RODRÍGUEZ-ESPINOSA, JHON-ALEXANDER. Ácidos ganodéricos en Ganoderma $s p$, Ganoderma lucidum y en su sustrato agotado. Biotecnología en el sector agropecuario y agroindustrial, v. 19, n. 1, 2021. DOI: https://doi.org/10.18684/BSAA(19)206-216 


\section{RESUMEN}

Los hongos del Género Ganoderma son reconocidos mundialmente por sus propiedades medicinales y su mercado nacional e internacional crece continuamente. Con el objetivo de indagar acerca del potencial comercial de nuestra diversidad fúngica, se determinaron los contenidos de compuestos bio-activos, conocidos como Ácidos Ganodéricos (A.G.), a partir de tres fuentes del hongo. De los cuerpos fructíferos de dos variedades de Ganoderma sp y Ganoderma lucidum (silvestre y comercial). La variedad silvestre fue recolectada en el municipio de Quimbaya Quindío, la comercial, certificada, se cultivó sobre residuos agroindustriales de plátano y el sustrato agotado fue obtenido después de la recolección de la variedad comercial. Las tres muestras obtenidas por triplicado, fueron secadas y sometidas a extracción con fluidos supercríticos y por ultrasonido. La concentración de A.G. se determinó por Cromatografía Líquida (HPLC), por el método de normalización de áreas, usando como estándar el Acido Ganodérico A. Los mayores rendimientos de extracción fueron obtenidos por el método de ultrasonido, independientemente de la muestra analizada. El mayor contenido de A.G., fue encontrado en la cepa silvestre (266 $\mu \mathrm{g} / \mathrm{g})$, aproximadamente $30 \%$ más que en la variedad comercial, aunque considerablemente menor a estudios internacionales. Es posible concluir que la variedad silvestre tendría potencial para convertirse en una fuente comercial de A.G.

\section{ABSTRACT}

Ganoderma genus are recognized worldwide for their medicinal properties and their national and international market is continuously growing. In order to investigate the commercial potential of our fungal diversity, the contents of bioactive compounds, known as Ganodic Acids (A.G.), were determined from three sources of fungus. Of the fruiting bodies of two varieties of Ganoderma sp and Ganoderma lucidum (wild and commercial). The wild variety was collected in the municipality of Quimbaya Quindío, the commercial, certified, was developed on agro-industrial plantain waste and the spent substrate were obtained after the harvesting of the commercial variety. The three samples obtained in triplicate, were dried and subjected to extraction with supercritical fluids and ultrasound. The concentration of A.G. was determined by Liquid Chromatography (HPLC), by the method of normalization of areas, using as standard Gano$\operatorname{deric}$ Acid A. The method of extraction by ultrasound was the most efficient in all cases. The highest content of A.G., was found in the wild strain $(266 \mu \mathrm{g} / \mathrm{g})$, approximately $30 \%$ more than in the commercial variety, although considerably less than international studies. It is possible to conclude that the wild variety would have the potential to become a commercial source of A.G.

\section{RESUMO}

Os fungos do gênero Ganoderma são reconhecidos mundialmente por suas propriedades medicinais e seu mercado nacional e internacional está crescendo continuamente. A fim de investigar o potencial comercial da nossa biodiversidade fúngica, o conteúdo de compostos bioativos, conhecidos como Ácidos Ganódericos (A.G.), foi determinado a partir de três fontes de fungos.

\section{PALABRAS CLAVE:}

Cultivo; Basidiomicetos; Cepa nativa; Extracción; Metabolitos secundarios; Cromatografía; Ganoderma lucidum.

\section{KEYWORDS:}

Culture; Basidiomycetes; Wild strain; Extraction; Secondary metabolites; Chromatography; Ganoderma lucidum.

\section{PALABRAS-CHAVE:}

Cultura; Basidiomiceto; Linhagem nativa; Extração; Metabolitos secundários; Cromatografia; Ganoderma lucidum. 
Dos corpos frutíferos de duas variedades de Ganoderma sp e Ganoderma lucidum (silvestre e comercial). A variedade silvestre foi coletada no município de Quimbaya Quindío, a comercial, certificada, foi desenvolvida sobre resíduos de bananeira agroindustrial e do substrato gasto obtido após a colheita da variedade comercial. As três amostras obtidas em triplicata, foram secas e submetidas à extração com fluidos supercríticos e ultra-som. A concentração de A.G. foi determinado por Cromatografia Líquida (HPLC), pelo método de normalização de áreas, utilizando como padrão o Ácido Ganodérico A. O método de extração por ultrassom foi o mais eficiente em todos os casos. O maior teor de A.G., foi encontrado na estirpe selvagem ( $266 \mu \mathrm{g} / \mathrm{g})$, aproximadamente $30 \%$ mais do que na variedade comercial, embora consideravelmente menos do que os estudos internacionais. É possível concluir que a variedade silvestre teria o potencial de se tornar uma fonte comercial de A.G.

\section{INTRODUCCION}

El hongo Ganoderma lucidum, Lingzhi o Mannetake es conocido en la cultura China y japonesa, desde hace más de 2000 años y se consume con frecuencia, ya que además de ser fuente de nutrientes, tiene propiedades nutracéuticas. En las últimas décadas se han acumulado pruebas científicas sobre la aplicación médica del hongo en el tratamiento de varios tipos de dolencias como bronquitis, anorexia, gastritis, hepatitis, nefritis, hemorroides, dismenorrea, estreñimiento, lupus, enfermedades crónicas como migraña, hipertensión, artritis, asma, diabetes, hipercolesterolemia, enfermedades cardiovasculares, e inclusive el cáncer [1]. Los compuestos bio-activos a los que se les atribuyen estas propiedades son los Ácidos Ganodéricos (A.G.) y los Beta-glucanos, entre otros [2].

Actualmente, los hongos del género Ganoderma, son utilizados, no solo para el consumo en fresco o seco, sino, además para la producción de diversos productos alimenticios funcionales y nutracéuticos [3,4,5].

En Colombia y en el resto del mundo, continuamente, se realizan cultivos con cepas certificadas, sobre residuos agroindustriales, específicos de cada región, buscando encontrar las formulaciones que permitan los mayores rendimientos de producción y concentraciones de compuestos bio-activos, como los A.G. [6,7].

En el Departamento del Quindío se han encontrado cepas silvestres de Ganoderma, adaptadas a nuestros ecosistemas y que pueden contener los compuestos bio-activos en concentraciones que pueden llegar a ser de interés comercial.

Por las anteriores razones, es de gran importancia estudiar en términos de análisis cualitativos y cuantitativos, la presencia y cantidad de los compuestos bio-activos de Ganoderma, presentes en cepas silvestres y comerciales, e inclusive en la biomasa, donde se han desarrollado estos hongos (sustrato agotado o residual) $[8,9,10,11,12,13]$.

Varios métodos de extracción con solventes, han sido desarrollados con el propósito de obtener los compuestos bio-activos del género Ganoderma [14,15,16], siempre buscando aumentar los rendimientos para desarrollar futuras aplicaciones comerciales, disminuir los costos y evitar la degradación de las sustancias de interés.

La extracción con fluido supercrítico (SFC), es una técnica que ha permitido la extracción, de compuestos bio-activos de Ganoderma, utilizando bajas temperaturas y altas presiones [17, 18, 19]. Además, por su reducido consumo y bajo costo de solventes, manejo automatizado de la muestra y alta eficiencia, se convierte en una alternativa viable en éste tipo de investigaciones [20]. De forma alternativa, la técnica de ultrasonido o sonicación intensa, se ha evaluado [21,22], en este caso, con el fin de extraer ácidos Ganodéricos, la membrana celular debe ser destruida. Para la disrupción, se requiere encontrar un procedimiento adecuado de extracción y así lograr la liberación de todos los productos [23].

Los Ácidos Ganodéricos pueden ser separados, identificados y cuantificados, utilizando la técnica de Cromatografía Líquida (HPLC) $[24,25,26)$, acoplada a detector UV-Vis. Está técnica es ampliamente usada, por su rapidez y eficiencia, dado que mediante la comparación con estándares externos e internos, es posible la identificación y cuantificación de las sustancias presentes. 
En ésta investigación se comparó la técnica de extracción con fluido supercrítico (SFC) con la técnica de extracción con ultrasonido (EUA), tomando como base la concentración de Ácidos Ganodéricos obtenidos al final del proceso.

En ésta investigación, se extrajeron los Ácidos Ganodéricos, presentes en el cuerpo fructífero y el sustrato agotado del hongo Ganoderma lucidum, mediante las técnicas de extracción con fluido supercrítico (SFC) y extracción con ultrasonido (EUA). Se identificaron y cuantificaron mediante la cromatografía líquida (HPLC), acoplada a espectrofotometría UV-Vis.

\section{MÉTODO}

\section{Obtención de Ganoderma sp}

Los cuerpos fructíferos de Ganoderma sp, fueron colectados cerca de la reserva el ocaso $\left(4^{\circ} 58^{\prime} 73.8^{\prime \prime} \mathrm{N}\right.$ 7585'52.4”W), en el municipio de Quimbaya, Quindío.

\section{Semilla de Ganoderma lucidum comercial}

Para la siembra de G. lucidum sobre residuos de plátano, se utilizó una semilla certificada, proporcionada por la planta de bio-procesos y agroindustria de la Universidad de Caldas. Esta semilla fue obtenida por inoculación del micelio de G. lucidum sobre cebada

\section{Cultivo de G. Iucidum}

Como sustrato para la producción, se utilizaron subproductos del monocultivo de plátano (pseudotallos, hojas y raquis), fraccionados en cortes de aproximadamente $1 \mathrm{~cm}^{2}$, esterilizados y formulados para obtener relaciones de Carbono/Nitrógeno del orden de 40 a 60 . El proceso se llevó a cabo en la granja Bengala $\left(4^{\circ} 41^{\prime} 08.8^{\prime \prime} \mathrm{N}\right.$ $75^{\circ} 37^{\prime} 02.9^{\prime \prime} \mathrm{W}$ ), propiedad de la Universidad del Quindío, ubicada en el municipio de Finlandia (Quindío). Después de 38 semanas de crecimiento, fueron recolectados los cuerpos fructíferos y además el sustrato agotado.

\section{Tratamiento de la muestra}

Los cuerpos fructíferos de G. lucidum cultivado sobre residuos de plátano, de la especie Ganoderma sp y el sustrato agotado, se secaron y deshidrataron a $40^{\circ} \mathrm{C}$ por 24 horas; de allí, se tomaron por separado para ser triturados, utilizando un molino convencional y luego fueron tamizados hasta alcanzar la pulverización homogénea en un tamaño de $50 \mu \mathrm{m}$, de forma similar a lo reportado por algunos autores [1,27]

\section{Extracción con fluido supercrítico (SFC)}

Este procedimiento, se llevó a cabo en el instituto de Biotecnología y Agroindustria de la Universidad Nacional de Manizales. Se utilizó solvente supercrítico $\left(\mathrm{CO}_{2}\right)$ y solvente etanol, a 300 bar de presión, durante 1 hora y con temperatura de $40^{\circ} \mathrm{C}$, secuencialmente controlados [28]. Se colocaron $15 \mathrm{~g}$ de muestra pulverizada en la cámara de extracción del reactor dentro de una funda de lienzo. Fueron obtenidos 6 extractos, pertenecientes a cada una de las muestras por duplicado

\section{Extracción con ultrasonido (EUA)}

Fue realizada en el laboratorio de catálisis de la universidad del Quindío en un sonicador BRANSON 1800 de 45 $\mathrm{MHz}$, durante 30 minutos [30]. Se depositaron $2 \mathrm{~g}$ del pulverizado y se disolvieron en $20 \mathrm{~mL}$ de etanol de $95 \%$, contenidos en un vaso de precipitado de $50 \mathrm{~mL}$. Se dejaron reposar durante 24 horas, luego, de la parte sobrenadante de cada uno, se filtró $1 \mathrm{~mL}$ dentro de los viales para HPLC, usando PVDF de 0,45 $\mu \mathrm{m}$ Millipore millex-HV. 


\section{Preparación del estándar y adición a los extractos}

5 mg del estándar de Ácido Ganodérico A, (SIGMA-ALDRICH®) se disolvieron en $5 \mathrm{~mL}$ de etanol grado HPLC. De esta disolución madre, se preparó una disolución a $50 \mathrm{\mu g} / \mathrm{mL}$, usando etanol como disolvente. A cada uno de los extractos, debidamente diluidos, para conservar las proporciones, se les adicionó una concentración fija de Ácido Ganodérico A.

\section{Cromatografía y cuantificación}

Los extractos fueron inyectados en el cromatógrafo líquido Thermo Dionex 3000 , en las condiciones que a continuación se describen: Columna de separación ACE AQ C-18 (250 mm x 4,6 mm ID, 5 micras), y fase móvil de ácido fórmico al 0,5\% y acetonitrilo en proporciones 60 (A) y 40 (B) en modo isocrático. Se utilizó un sistema de detección UV-Vis con arreglo de diodos (DAD), a 250 y 280 nm. La cuantificación de los Ácidos Ganodéricos se realizó por el método de normalización de áreas, comparando el área del Ácido Ganodérico A (de concentración conocida), con el área de los picos de Ácidos Ganodéricos, presentes en la muestra.

\section{Identificación Ganoderma sp}

Aunque de éste hongo se conocen más de 250 especies reportadas en todo el mundo, la cepa silvestre pudo ser clasificada usando la metodología empleada en la taxonomía tradicional, al tener en cuenta el análisis de las características morfológicas del cuerpo fructífero recolectado.

La clave taxonómica que se tuvo en cuenta fue reportada por Weber en 2009 [31]. En éste género, se pudo observar la presencia de un píleo o sombrero en forma de repisa mientras que la parte inferior del esporocarpo presenta una multitud de poros que ratifican de que se trata de una especie polyporaceae (Figura 1). Además, el olor a madera añejada y su color café brillante, son característicos del género, por lo que se pudo clasificar ésta cepa silvestre como Ganoderma sp.

Figura 1. Cuerpo fructífero de Ganoderma sp.

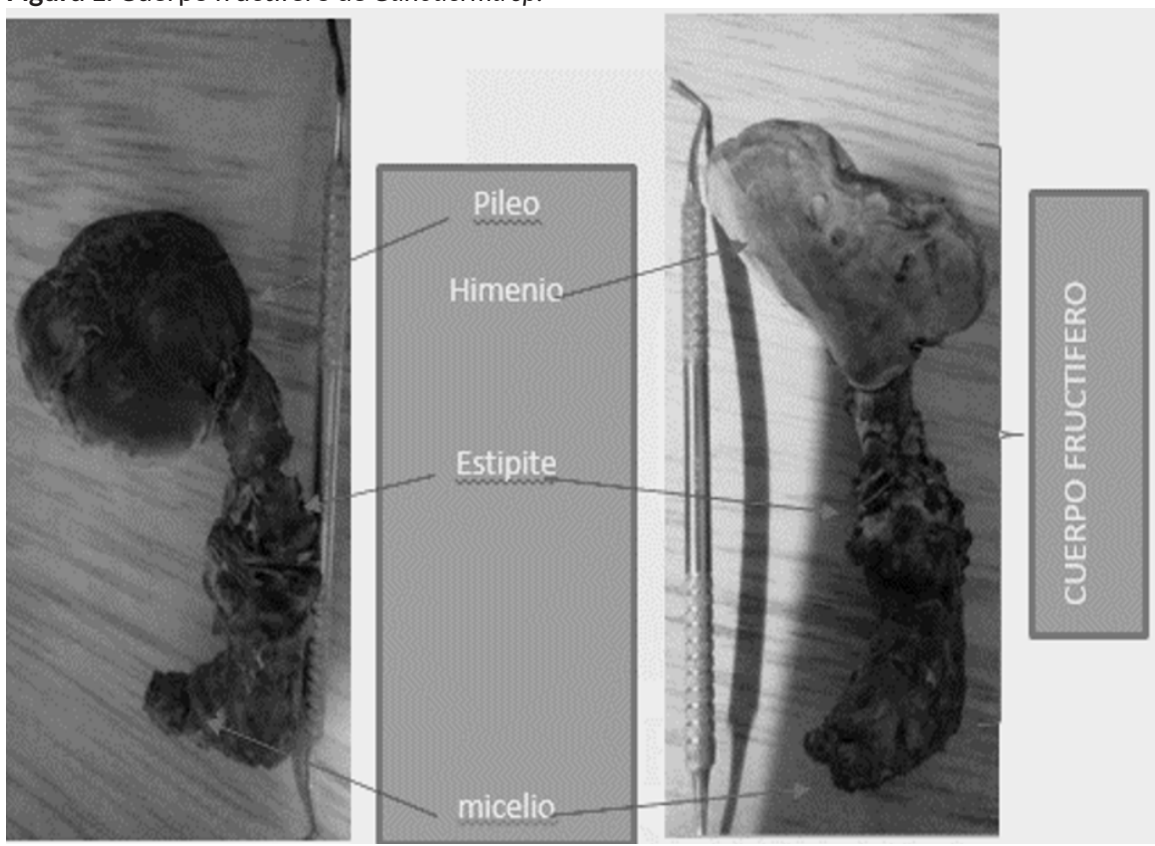

Fuente: Wilder Ramírez 


\section{Análisis cromatográfico}

Con el método cromatográfico utilizado, se lograron aislar y detectar dos señales o picos principales (Figura 2). La primera de ellas corresponde al Ácido Ganodérico A, utilizado como estándar, con un tiempo de retención de 7,48 min, que fue confirmada con una corrida cromatográfica del estándar en forma aislada.

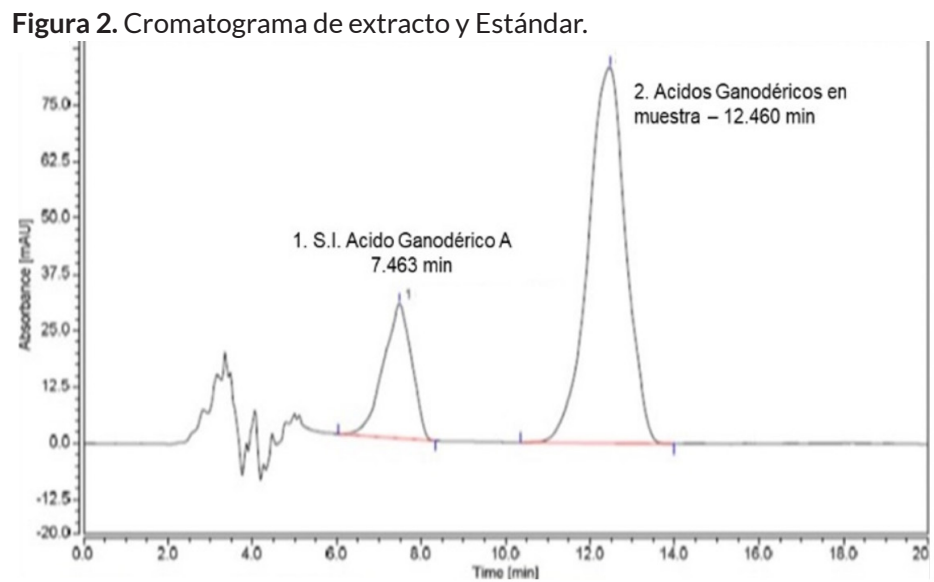

La segunda señal corresponde a los Ácidos Ganodéricos presentes en los extractos de las muestras, con un tiempo de retención de 12,45 min. Posterior a éste tiempo no se observan nuevas señales.

Se observa un solapamiento de picos o co- elución en la señal, que aparece de mayor tamaño. Las condiciones del método no fueron suficientes para obtener una mejor resolución y separar cada uno de los Ácidos Ganodéricos presentes en el extracto, posiblemente debido a la composición de la fase estacionaria de la columna cromatográfica..

A pesar de que no fue posible separar los Ácidos Ganodéricos presentes en el extracto, se corroboró su presencia, tomando el espectro UV-Vis del pico a 12,45 min y comparándolo con aquellos reportados en la bibliografía [32]. Con la ayuda del software del detector UV-Vis con arreglo de diodos, se pudo determinar también, el solapamiento de los picos en la señal. Tal como se observa en la Figura 3.

Figura 3. Verificación del solapamiento de picos de los Ácidos Ganodéricos.

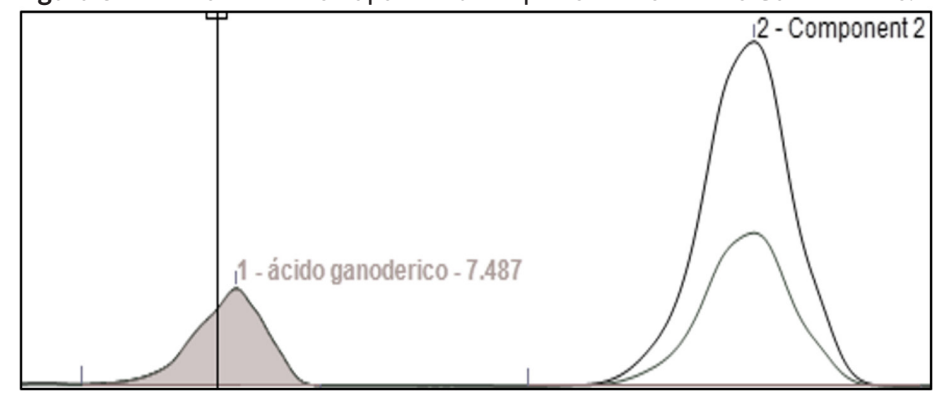

Tanto los espectros y los tiempos de retención, indican que la señal producida se debe a los Ácidos Ganodéricos $\mathrm{B}$ y $\mathrm{H}$. Estos ácidos tienen una gran semejanza estructural (son isómeros). 
Liu et al. (2011), investigaron condiciones cromatográficas semejantes y encontraron que los Ácidos Ganodéri$\cos \mathrm{B}$ y $\mathrm{H}$ aparecieron en un pico superpuesto, probablemente por la determinación concomitante de los Ácidos Ganodéricos $\mathrm{B}$ y $\mathrm{H}$; es decir, que la orientación espacial de los radicales o los sustituyentes $\mathrm{R}_{1}, \mathrm{R}_{2}, \mathrm{R}_{3}$ y $\mathrm{R}_{4}$ tendrían una afinidad análoga por la fase estacionaria [29]. La figura 4 y el cuadro 1, presentan la similitud estructural de los ácidos Ganodéricos, tomando como base información científica actualizada.

Figura 4. Estructura química de los Ácidos Ganodéricos.

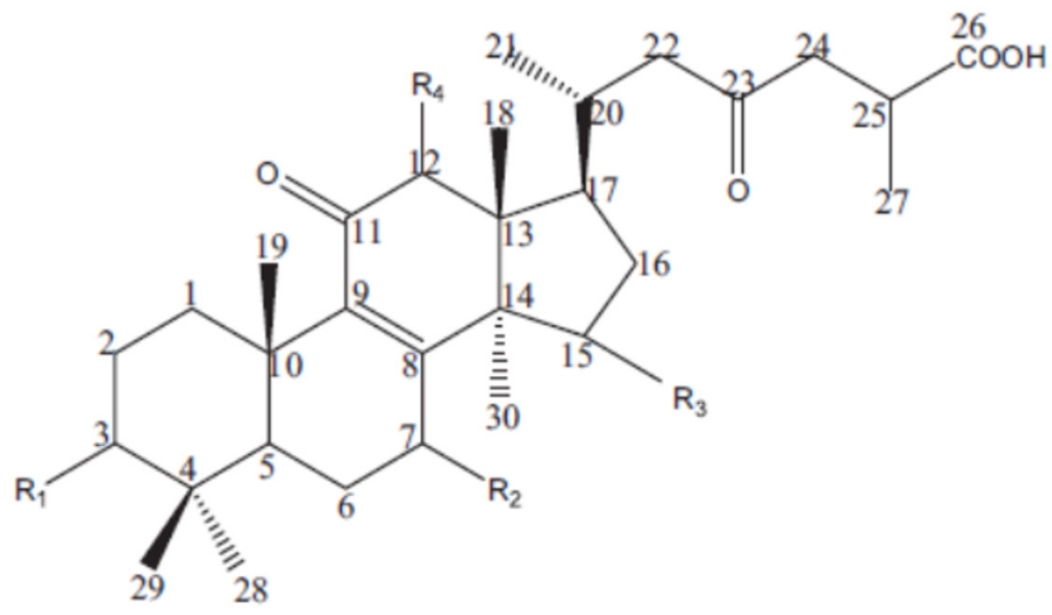

Tomado de: http://www.plantasyhongos.es/herbarium/htm/Ganoderma_lucidum.htm

Cuadro 1. Sustituyentes, presentes en los Ácidos Ganodéricos.

\begin{tabular}{|c|c|c|c|c|}
\hline Ácido Ganodérico & $\mathbf{R}_{1}$ & $\mathbf{R}_{2}$ & $\mathbf{R}_{3}$ & $\mathbf{R}_{4}$ \\
\hline $\mathrm{A}$ & $=\mathrm{O}$ & $\beta-\mathrm{OH}$ & $\alpha-\mathrm{OH}$ & $-\mathrm{H}$ \\
\hline $\mathrm{B}$ & $\beta-\mathrm{OH}$ & $\beta-\mathrm{OH}$ & $=\mathrm{O}$ & $-\mathrm{H}$ \\
\hline $\mathrm{H}$ & $\beta-\mathrm{OH}$ & $=\mathrm{O}$ & $=\mathrm{O}$ & $\beta-\mathrm{OAc}$ \\
\hline
\end{tabular}

Tomado de: http://repositoriodigital.uns.edu.ar/bitstream/123456789/3786/1/Tesis\%20 Maximiliano\%20Bidegain\%20-\%20corregida\%20final.pdf

\section{Cuantificación de los Ácidos Ganodéricos}

El análisis cuantitativo de los extractos del sustrato agotado y Ganoderma sp, muestra que la técnica de extracción por ultrasonido (EUA) es más eficiente que la técnica de extracción por fluidos supercríticos (SFC), en cuanto a la concentración de Ácidos Ganodéricos obtenidos. En estos casos, las concentraciones fueron: 161,19 g/g para el sustrato agotado y $265,85 \mathrm{\mu g} / \mathrm{g}$ para Ganoderma silvestre cuando se utilizó extracción con ultrasonido (EUA) y $146,66 \mu \mathrm{g} / \mathrm{g}$ para el sustrato agotado y 181,52 $\mu \mathrm{g} / \mathrm{g}$ para Ganoderma cuando se utilizó extracción por fluidos supercríticos (SFC).

Para el caso de la extracción de Ácidos Ganodéricos a partir del cuerpo fructífero de la cepa comercial Ganoderma lucidum, se observan concentraciones similares en ambas técnicas de extracción, con 182,47 $\mu \mathrm{g} / \mathrm{g}$ utilizando EUA y $186,19 \mu \mathrm{g} / \mathrm{g}$, utilizando SFC.

La mayor concentración de Ácidos Ganodéricos se presenta en los extractos de Ganoderma sp, seguida de Ganoderma lucidum comercial, independientemente del método de extracción empleado (Figura 5). 
Figura 5. Comparación de las concentraciones de Ácidos Ganodéricos.

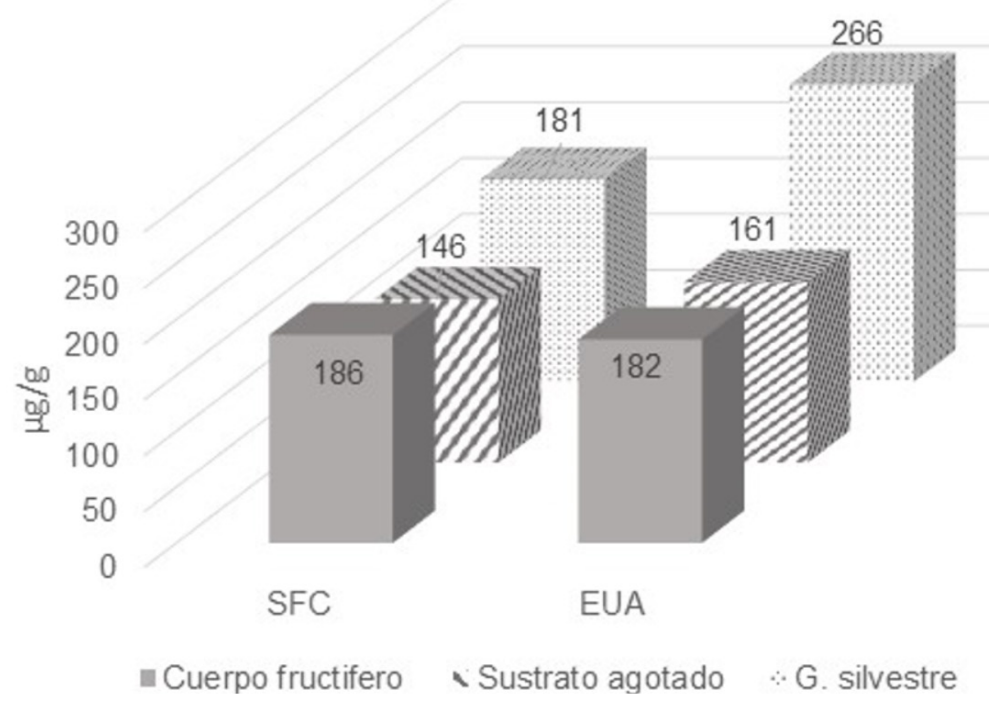

SFC: Extracción por fluidos supercríticos.

EUA: Extracción por Ultrasonido

Los rangos de concentraciones de Ácidos Ganodéricos que se obtuvieron en ésta investigación (entre 266 y 147 $\mu \mathrm{g} / \mathrm{mL}$ ) se pueden comparar con los de otros autores [1, 1529,30$]$. De acuerdo a los mostrado en la figura 6 , las concentraciones reportadas en éste estudio, varían o fluctúan entre los rangos reportados en otros artículos.

Figura 6. Ácidos Ganodéricos encontrados en éste estudio vs otras investigaciones.

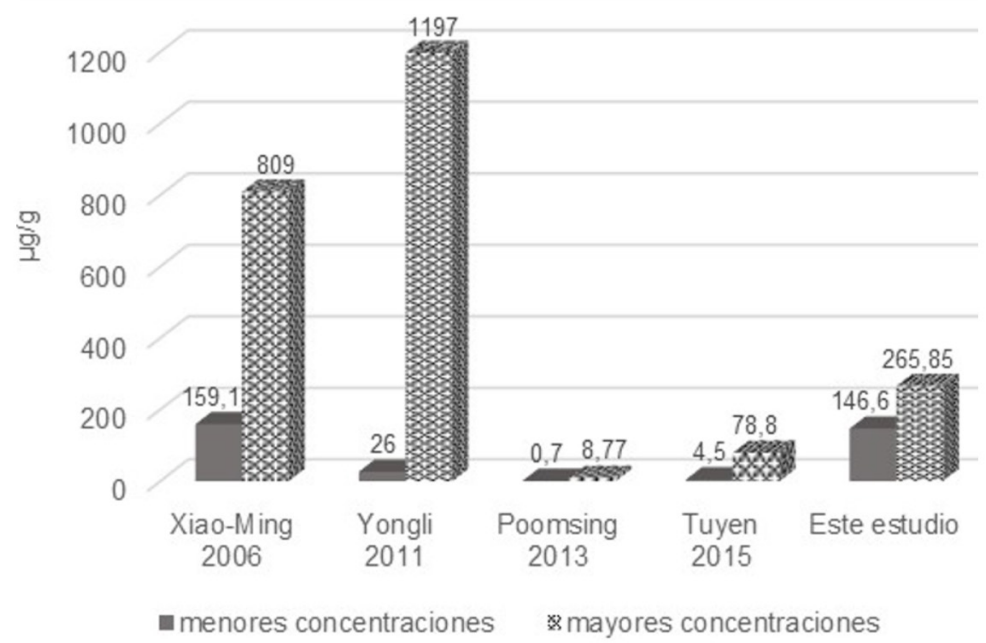

La mayor concentración de Ácidos Ganodéricos, fue reportada por Nguyen y Yongli en 2011, con 1197 mg/mL pero cabe resaltar que fueron utilizadas las esporas para éste estudio y no el cuerpo fructífero [15]. De igual forma Xiao-Ming Wang et al (2006) [30] reportaron concentraciones de Ácidos Ganodéricos B y H en Ganoderma lucidum. Para el Acido Ganodérico B, desde 32 a 165 ug/mL y para el Ácido Ganodérico H desde 127,1 hasta $643,9 \mu \mathrm{g} / \mathrm{mL}$, que para efectos prácticos fueron sumadas y aparecen en la gráfica 6 como una sola barra. Los resultados se obtuvieron de los extractos de cinco especies diferentes de Ganoderma originarias de China, usando etanol de $95 \%$, un calentador de inmersión y concentrados por combinaciones de agua caliente, bases y ácidos 
Los resultados de la figura 6 permiten dar una visión de la incidencia de los métodos de extracción, la parte del hongo a estudiar y la especie, sobre la concentración de Ácidos Ganodéricos que pueden ser obtenidos. Se observan diferencias muy significativas de concentraciones debido a los factores anteriormente nombrados.

\section{CONCLUSIONES}

Enésta investigación, se pudo comparar el efecto de dos métodos extracción sobre diferentes muestras de Ganoderma.

Adicionalmente se determinaron las condiciones cromatográficas, para la cuantificación rápida de Ácidos Ganodéricos (A.G.) presentes en las muestras. Las concentraciones de A.G encontradas. se ubicaron en los rangos publicados por otros autores

\section{AGRADECIMIENTOS}

Ricardo Soto, Docente del programa de Biología, MSc en Medio Ambiente. Por su ayuda en la recolección e identificación del Ganoderma sp

\section{REFERENCIAS}

[1] POOMSING, P.; PATTANAPANYASAT, K.; WONGSINKONGMAN, P.; SOONTHORNCHAREONNON, N. Research and Development of Ganoderma lucidum Cultivated in Thailand. Journal of Pharmaceutical Sciences, v. 40, n. 3, 2013, p.1-7.

[2] BABY, SABULAL; JOHNSON, ANIL-JOHN; GOVINDAN, BALAJI. Secondary metabolites from Ganoderma. Phytochemistry, v. 114, 2015, p. 66-101. https://doi.org/10.1016/j.phytochem.2015.03.010

[3] BISHOP, KAREN; KAO, CHI-HSIU; XU, YUANYE; GLUCINA, MARCUS P.; PATERSON, RUSELL R.; FERGUSON, LYNNETTE R. From 2000 years of Ganoderma lucidum to recent developments in nutraceuticals. Phytochemistry, v. 114, 2015, p. 56-65. https://doi.org/10.1016/j.phytochem.2015.02.015

[4] TAOFIQ, OLUDEMI; HELENO, SADRINO; CALHELHA, RICARDO; ALVES, MARÍA-JOSÉ; BARROS, LILLIAM; GONZÁLEZ-PARAMÁS, ANA-MARÍA; BARREIRO, MARIA F.; FERREIRA, ISABELL. The potential of Ganoderma lucidum extracts as bioactive ingredientes in topical formulations, beyond its nutritional benefits. Food and Chemical Toxicology, v. 108, n. A, 2017, p. 139-147. https://doi.org/10.1016/j.fct.2017.07.051

[5] TAOFIQ, OLUDEMI; GONZÁLEZ-PARAMÁS, ANA-MARIA; MARTINS, ANABELA; BARREIRO, MARIA-FILOMENA; FERREIRA, ISABELL. Mushrooms extracts and compounds in cosmetics, cosmeceuticals and nutricosmetics-A review. Industrial Crops and Products, v. 90, 2016, 38-48.

[6] POSTEMSKY, PABLO; BIDEGAIN, MAXIMILIANO; GONZALEZ-MATUTE, RAMIRO; FIGLAS, N.D.; CUBITTO, M.A. Pilot-scale bioconversion of rice and sunflower agro-residues into medicinal mushrooms and laccase enzymes through solid-state fermentation with Ganoderma lucidum. Bioresource Technology, v. 231, 2017, p.85-93. https://doi.org/10.1016/j.biortech.2017.01.064

[7] BINGZHI, CHEN; BINRONG, K.E.; LIYUN, Y.E.; SHANSHAN, JIN; FAN, J.I.E. Isolation and varietal characterization of Ganoderma resinaceum from areas of Ganoderma lucidum production in China. Scientia Horticulturae, v. 224, 2017, p. 109-114.

[8] POSTEMSKY, PABLO D.; MARINANGELI, PABLO A.; CURVETTO, NESTOR-RAÚL. Recycling of residual substrate from Ganoderma lucidum mushroom cultivation as biodegradable containers for horticultural seedlings. Scientia Horticulturae, v. 201, 2016, 329-327.

https://doi.org/10.1016/j.scienta.2016.02.021 
[9] OZCARIZ-FARMOSELLE, MARIA-VIRGINIA; FRAILE-FABERO, RAÚL; GIRBÉS,JUAN- TOMAS; ARCE-CERVANTES, OSCAR; ORIA DE RUEDA-SALGUEIRO, JUAN-ANDRES; AZUL, ANABELA-MARISA. Use of lignocellulosic waste of pecan (Carya illinoinensis) in the cultivation of Ganoderma lucidum. Revista Iberoamericana de Micología, v. 35, n. 2, 2018, p. 103-109.

[10] XINGRONG, PENG; LEI, LI; JINRUN, DONG; SHUANGYANG, LU; JING, UL; XIAONIAN, LI; MINGHUA, QIU. Lanostane-type triterpenoids from de fruiting bodies of Ganoderma applanatum. Phytochemistry, v. 157, 2019, p. 103-110. https://doi.org/10.1016/j.phytochem.2018.10.011

[11] CHENLEI, LIAN; CUIFANF, WANG; QIN, XIAO; LEN, XIAO; YING, SU; JIEQUING, LU. The triterpenes and steroids from the fruiting body Ganoderma duripora. Biochemical Systematics and Ecology, v. 73, 2017, p.50-53. https://doi.org/10.1016/j.bse.2017.06.005

[12] XIAN-QIANG, CHEN; JING, ZHAO; LING-XIAO, CHEN; SHEN-FEI, WANG; YING, WANG; SHAO-PING, LI. Lanostane triterpenes from the mushroom Ganoderma resinaceum and their inhibitory activities against a-glucosidase. Phytochemistry, v. 149, 2018, p. 103-115. https://doi.org/10.1016/j.phytochem.2018.01.007

[13] ZHANG, JIANJUN; GUANGYUAN, MENG; ZHAI, GUOYIN; YANG, YONGHENG; ZHAO, HUAJIE; JIA, LE. Extraction, characterization and antioxidant activity of polysaccharides of spent mushroom compost of Ganoderma lucidum. International Journal of Biological Macromolecules, v. 82, 2016, p. 432-439. https://doi.org/10.1016/j.ijbiomac.2015.10.016

[14] SALGADO-ORDOSGOITIA, RODRIGO-DANIEL; ARIAS-MARTÍNEZ, JOHANNA-MILENA; ACOSTA-CHÁVEZ, AMPARO-DEL CRISTO; RODRÍGUEZ-MANRIQUE, JHONATAN-ANDRÉS; PÉREZ-PÉREZ, MARIO-FRANK. Chemical study of fraction ethyl acetate in the fungus Ganoderma lucidum, grown in agro-industrial waste. Revista de la academia colombiana de ciencias exactas, físicas y naturales, v. 40, n. 157, 2016, p. 600-607. https://doi.org/10.18257/raccefyn.391

[15] NGUYEN, M.T.; NGUYEN, T.M. Extraction of bioactive compounds and spore powder collection from Ganoderma lucidum. Can tho University Journal of Science, v. 1, 2015, p. 53-60.

[16] SOOD, GUNJAN; SHARMA, SHIVANI; KHANNA, P.K.; KAPOOR, S. Optimization of extraction and characterization of polysaccharides from medicinal mushroon Ganoderma lucidun using response surfase methodology. Journal of Medicinal Plants reseach, v. 7, n. 31, 2013, p. 2323-2329. https://doi.org/10.5897/JMPR2013.5115

[17] RUHAN-ASKIN, UZEL; UNAL-RIZA, YAMAN. A novel method for food particle production using subcritical water extraction: Ganoderma mushroom as a case example. The Journal of Supercritical Fluids, v. 111, 2016, p. 74-82. https://doi.org/10.1016/j.supflu.2016.01.021

[18] LIAN, LI; HUI-JUN, GUO; LING-YANG, ZHU; LIMIN, ZHENG; XIN, LIU. A supercritical-CO ${ }_{2}$ extract of Ganoderma lucidum spores inhibits cholangiocarcinoma cell migration by reversing the epithelial-mesenchymal transition. Phytomedicine, v. 23, 2016, p. 491-497. https://doi.org/10.1016/j.phymed.2016.02.019

[19] JINWEI, LI; XINXIN, ZHANG; YUANFA, LIU. Supercritical carbon dioxide extraction of Ganoderma lucidum spore lipids. LWT-Food Science and Technology, v. 70, 2016, p. 16-23. https://doi.org/10.1016/j.lwt.2016.02.019

[20] MEIRELES, A.; CARVALHO, RAÚL; MOURA, LUCINEWTON; ROSA, PAULO. Supercritical fluid extraction from rosemary (Rosmarinus officialis): Kinetic data, extract's global yield, composition, and antioxidant activity. The Journal of Supercritical Fluid, v. 35, 2005, p. 197-204. https://doi.org/10.1016/j.supflu.2005.01.009

[21] QIAOZHEN, KANG; SISI, CHEN; SHUFANG, LI; BO, WANG; XIN, LIU; LIMIN, HAO; JIKE, LU. Comparison on characterization and antioxidant activity of polysaccharides from Ganoderma lucidum by ultrasound and conventional extraction. Internacional Journal of Biological Macromolecules, v. 124, 2019, p. 1137-1144. https://doi.org/10.1016/j.ijbiomac.2018.11.215 
[22] ALZORQI, IBRAHIM; SINGH, AJIT; MANICKAM, SIVAKUMAR; AL-QRIMLI, F. Optimization of ultrasound assisted extraction (UAE) of $\beta$-D-glucan polysaccharides from Ganoderma lucidum for prospective scale-up. Resource efficient technologies, v. 3, 2017, p. 46-54. https://doi.org/10.1016/j.reffit.2016.12.006

[23] AZUOLA, R.; VARGAS-AGUILAR, P. Extracción de sustancias asistida por ultrasonido (EUA). Revista Tecnología en marcha, v. 20, 2007, p. 30-40.

[24] RAMAKRISHNA, M.B; BADU, RAJESH.; VEENA, S.S; PANDEY, MEERA; RAO, NAGESWARA. Validated Reverse-Phase HPLC Method for Quantitative Determination of Ganoderic Acids A and B in Cultivated Strains of Ganoderma spp. (Agaricomycetes) Indigenous to India. International Journal of Medicinal, v. 19, 2017, p. 457-465.

[25] LINGXIAO, CHEN; XIANQIANG, CHEN; SHENFEI, WANG; YAO,BIAN; JIN,ZHAO; SHAOPING, LI. Analysis of triterpenoids in Ganoderma resinaceum using liquid chromatography coupled with electrospray ionization quadrupole time of flight mass spectrometry. International Journal of Mass Spectrometry, v. 436, 2019, p.42-51. https://doi.org/10.1016/j.ijms.2018.11.016

[26] LIU, WEI; ZHANG, JINGSONG; HAN, WEI; LIU, YANFANG; FENG, JIE; TANG, CHUANHONG; FENG, NA; TANG, QINGJIU. One single standar substance for the simultaneous determination of 17 triterpenes in Ganoderma lingzhi and its related species using high-performance liquid chromatography. Journal of Cromatography, v. 1068-1069, 2017, p. 49-55. https://doi.org/10.1016/j.jchromb.2017.10.010

[27] TI-QIANG, CHEN; XIAO-YAN, ZHAO; JIN-ZHONG, WU; DE-YI, YU; YAN-BING, WU. Supercritical fluid $\mathrm{CO}_{2}$ extraction, simultaneous determination of components in ultra-fine powder of Ganoderma sinense by HPLC-ESI-MS method. Journal of the Taiwan, v .42, 2011, p. 428-434. https://doi.org/10.1016/j.jtice.2010.08.003

[28] ZHAO, JING; XIAO-QI, ZHANG; SHAO-PING, LI; FENG-QING, YANG; YI-TAO, WANG; WEN-CAI, YE. Quality evaluation of Ganoderma through simultaneous determination of nine triterpenes and sterols using pressurized liquid extraction and high performance liquid chromatography. Journal of Separation Science, v. 17, 2006, p. 2609-2615. https://doi.org/10.1002/jssc.200600178

[29] LIU, YONGLI; LIU, YOUPING; QIU, FENG; DI, XIN. Sensitive and selective liquid chromatography-tandem mass spectrometry method for the determination of five ganoderic Acids in Ganoderma lucidum and its related species. Journal of Pharmaceutical and Biomedical Analysis, v. 54, 2011, p. 717-721. https://doi.org/10.1016/j.jpba.2010.11.002

[30] XIAO-MING, WANG; MIN, YANG; SHU-HONG, GUAN; RONG-XIA, LIU; JIA-MENG, XIA; KAI-SHUN, BI; DE-AN, GUO. Quantitative determination of six major triterpenoids in Ganoderma lucidum and related species by high performance liquid chromatography. Journal of Pharmaceutical and biomedical Analysis, v. 41, 2006, p. 838-844. https://doi.org/10.1016/j.jpba.2006.01.053

[31] WEBER, ROLAND. Recent developments in the molecular taxonomy of fungi. The mycota XV: Physiology and Genetics: Select Basic and Applied Aspects. Springer Heidelberg, p. 1-15

[32] SONIAMOL, JOSHEP; KAINOOR, JANARDHANAN; VARUGHESE, GEORGE; SABULAL, BABY. A new epoxidic ganoderic acid and other phytoconstituents from Ganoderma lucidum. Phytochemistry Letters, v. 4, n. 31, 2011, p. 386-398.

https://doi.org/10.1016/j.phytol.2011.08.011 